\title{
Chromo-Rayleigh interactions of dark matter
}

\section{Yang Bai and James Osborne}

Department of Physics, University of Wisconsin-Madison, 1150 University Ave, Madison, WI 53706, U.S.A.

E-mail: yangbai@physics.wisc.edu, jaosborne@wisc.edu

\begin{abstract}
For a wide range of models, dark matter can interact with QCD gluons via chromo-Rayleigh interactions. We point out that the Large Hadron Collider (LHC), as a gluon machine, provides a superb probe of such interactions. In this paper, we introduce simplified models to UV-complete two effective dark matter chromo-Rayleigh interactions and identify the corresponding collider signatures, including four jets or a pair of di-jet resonances plus missing transverse energy. After performing collider studies for both the $8 \mathrm{TeV}$ and $14 \mathrm{TeV}$ LHC, we find that the LHC can be more sensitive to dark matter chromo-Rayleigh interactions than direct detection experiments and thus provides the best opportunity for future discovery of this class of models.
\end{abstract}

Keywords: Beyond Standard Model, Cosmology of Theories beyond the SM

ARXIV EPRINT: 1506.07110 


\section{Contents}

1 Introduction 1

2 Contact interactions $\quad 2$

2.1 Thermal relic abundance 3

2.2 Direct detection 3

2.3 Collider constraints 4

3 Simplified UV-completion models $\quad 5$

3.1 QCD-charged particle mediation for $\mathcal{O}_{1}^{\text {cRayleigh }} \quad 6$

3.2 QCD-charged particle mediation for $\mathcal{O}_{2}^{\text {cRayleigh }} \quad 8$

$\begin{array}{lll}3.3 & \text { Pair-produced dijet resonances plus } E_{T}^{\text {miss }} & 11\end{array}$

4 Comparison to the direct detection limits 13

5 Discussion and conclusions 13

A Loop level calculations for the coefficient of $\mathcal{O}_{2}^{\text {cRayleigh }} \quad 14$

\section{Introduction}

Although there is no doubt that dark matter interacts gravitationally both among itself and with the Standard Model (SM) particles, we still have no convincing evidence for other dark matter interactions. Among the three forces in the SM, dark matter can not have order-one couplings under the electroweak forces. Otherwise, it will either emit photons or scatter off nuclei with a too-large cross section in direct detection experiments. Suppressed couplings to the $Z$ boson or the Higgs boson will be probed further in upcoming dark matter experiments [1]. For QCD interactions, the dark matter particle can not have a color charge due to confinement. If, instead, it is a QCD composite particle, the hadronic bound states should have a large interaction strength with SM pions and thus have a large scattering cross section with nucleons. This type of dark matter can not penetrate the Earth to reach detectors in underground direct detection experiments, but satellite-based X-ray quantum calorimetry experiments impose stringent bounds on this scenario [2].

Another way for dark matter to interact with gluons is through effective contact interactions, comparable to photon-molecule Rayleigh interactions. Introducing such higherdimensional effective operators to describe dark matter chromo-Rayleigh interactions is then independent of whether the dark matter is elementary or composite. For instance, in supersymmetric models the neutralino can couple to two gluons via a stop-top quark loop. In extra-dimensional models, a similar loop from the top quark and its Kaluza-Klein 
mode can generate the effective chromo-Rayleigh dark matter interaction [3]. Composite dark matter models can also generate chromo-Rayleigh interactions, with strengths typically suppressed by the composite scale [4]. In this paper, we perform a generic study on dark matter chromo-Rayleigh interactions. We pay particular attention to collider searches and the potential signatures associated with chromo-Rayleigh interactions. This study is similar to searches for electrical Rayleigh interactions of dark matter in ref. [5, 6], where the electrically charged particles can be searched for directly at the LHC.

For simplicity, we choose the dark matter particle to be a complex scaler denoted as $X$. We study two types of dimension-six interactions: $X^{\dagger} X G^{2}$ and $\left(X X-X^{\dagger} X^{\dagger}\right) G \widetilde{G}$, where $G$ is the QCD field tensor. For direct detection searches, the first operator provides spinindependent scattering while the second operator provides spin-dependent and momentumsuppressed scattering. For collider searches, the standard model independent signature is a mono-jet plus missing transverse energy. Taking into account the well-known limitations of such searches [7-24], we introduce simplified models to UV-complete these two operators. Collider signatures owing to such UV-completions depend strongly on how the colored mediators decay. We discuss several possible signatures, including multi-jets and pairproduced di-jet resonances with missing transverse energy.

The rest of this paper is organized as follows. In section 2, we work out the dark matter thermal relic abundance, direct detection cross sections, and collider constraints from the effective chromo-Rayleigh contact interactions. In section 3, we introduce two simplified models to UV-complete the previous contact operators. Section 3.1 introduces a scalar color-octet with the signature of four jets plus missing transverse energy, sections 3.2-3.3 introduce two fermion color-triplets with the additional signature of paired di-jet resonances plus missing transverse energy, and section 4 offers a comparison to direct detection experiments. We conclude in section 5 .

\section{Contact interactions}

Our model consists of a complex scalar dark matter field, $X$, which is a singlet under the SM gauge group. We introduce the following two $C P$-conserving, dimension six operators coupling dark matter to the gluon field

$$
\begin{aligned}
\mathcal{O}_{1}^{\text {cRayleigh }} & =\frac{\alpha_{s}}{4 \pi \Lambda_{1}^{2}} X^{\dagger} X G_{\mu \nu}^{a} G^{a \mu \nu}, \\
\mathcal{O}_{2}^{\text {cRayleigh }} & =\frac{i \alpha_{s}}{4 \pi \Lambda_{2}^{2}}\left(X X-X^{\dagger} X^{\dagger}\right) G_{\mu \nu}^{a} \widetilde{G}^{a \mu \nu},
\end{aligned}
$$

where $\Lambda_{i}$ is the cutoff scale and $\widetilde{G}^{a \mu \nu}=\frac{1}{2} \epsilon^{\mu \nu \alpha \beta} G_{\alpha \beta}^{a}$ is the dual gluon field strength tensor. The overall operator normalization accounts for a loop factor. In order for $X$ to be stable, we impose a $\mathcal{Z}_{2}$ symmetry under which $X$ is odd. Based on these two effective operators, we first calculate the thermal relic abundance, direct detection cross sections, and collider constraints. 


\subsection{Thermal relic abundance}

Depending on the UV physics, the dark matter sector could be more complicated than just one state. Therefore, the dark matter thermal relic abundance calculation based entirely on the operators in eqs. (2.1) and (2.2) can only provide a guidance for the potential parameter space in $M_{X}$ and $\Lambda_{i}$ for thermal dark matter. For the first operator, we have the dark matter self-annihilation rate from the process $X^{\dagger} X \rightarrow G G$ as

$$
\frac{1}{2}\left[\langle\sigma v\rangle\left(X^{\dagger} X \rightarrow G G\right)\right]=\frac{1}{2}\left[\frac{\alpha_{s}^{2}}{\pi^{3}} \frac{M_{X}^{2}}{\Lambda_{1}^{4}}\right] \equiv s,
$$

to leading order in the dark matter's relative velocity $v$ expansion. Here, the overall factor of $1 / 2$ is due to the relic density being comprised of particles and antiparticles. For the second operator, we have the annihilation rate from the process $X X\left[X^{\dagger} X^{\dagger}\right] \rightarrow G G$, in terms of components with $X=\left(X_{R}+i X_{I}\right) / \sqrt{2}$, as

$$
\frac{1}{2}\left[\langle\sigma v\rangle\left(X_{R} X_{I} \rightarrow G G\right)\right]=\frac{1}{2}\left[\frac{\alpha_{s}^{2}}{\pi^{3}} \frac{M_{X}^{2}}{\Lambda_{2}^{4}}\right] \equiv s .
$$

The dark matter relic abundance is inversely proportional to the annihilation rate and has a formula $\Omega_{X} h^{2} \approx 1.07 \times 10^{9} \mathrm{GeV}^{-1} x_{F} /\left(\sqrt{g^{*}} M_{\mathrm{pl}} s\right)$ with $g^{*}$ as the number of relativistic degrees of freedom at the freeze-out temperature and is taken to be 86.25 and the Planck scale is $M_{\mathrm{pl}}=1.22 \times 10^{19} \mathrm{GeV}$. The freeze-out temperature $x_{F}$ is given by $x_{F}=\ln \left[0.05 g M_{\mathrm{pl}} M_{X} s /\left(\sqrt{g^{*} x_{F}}\right)\right]$ with $g=2$ and is typically $\mathcal{O}(20)$.

\subsection{Direct detection}

For the first operator $\mathcal{O}_{1}^{\text {cRayleigh }}$, we can use the matrix element of $G_{\mu \nu}^{a} G^{a \mu \nu}$ inside a nucleon to derive the dark matter coupling to two nucleons. The trace anomaly of the QCD energymomentum tensor implies [25, 26]

$$
m_{N}\langle N \mid N\rangle=\left\langle N\left|\sum_{1 \leq i \leq n_{f}} m_{i} \bar{\psi}_{i} \psi_{i}(1+\gamma)+\left(\frac{\beta^{n_{f}}}{2 \alpha_{s}^{2}}\right) \alpha_{s} G_{\mu \nu}^{a} G^{a \mu \nu}\right| N\right\rangle,
$$

where $\beta^{n_{f}}=-\left(11-2 n_{f} / 3\right) \alpha_{s}^{2} / 4 \pi$ is the beta function at leading order, $n_{f}$ is the number of quarks, and $\gamma$ is the anomalous dimension of the quark field. So, at leading order in $\alpha_{s}$ and keeping only $n_{f}=3$ light quarks, we have

$$
\left\langle N\left|\alpha_{s} G_{\mu \nu}^{a} G^{a \mu \nu}\right| N\right\rangle=\frac{8 \pi}{9} m_{N}\left[\frac{1}{m_{N}}\left\langle N\left|m_{u} \bar{u} u+m_{d} \bar{d} d+m_{s} \bar{s} s\right| N\right\rangle-1\right] \equiv \frac{8 \pi}{9} m_{N}\left[\left(f_{u}+f_{d}+f_{s}\right)-1\right] .
$$

Recent Lattice QCD updates of the calculation for the strange quark matrix element has $f_{u}+f_{d}+f_{s}=0.085_{-0.014}^{+0.022}$ [27]. In our numerical calculation, we will use $\left\langle N\left|\alpha_{s} G_{\mu \nu}^{a} G^{a \mu \nu}\right| N\right\rangle \approx-2.56 m_{N}$. The formula of the scattering cross section is then calculated to be

$$
\sigma_{X N}^{\mathrm{SI}}=\frac{\kappa^{2} m_{N}^{4}}{4 \pi \Lambda_{1}^{4}\left(m_{N}+M_{X}\right)^{2}},
$$

with $\kappa=\frac{2}{9}\left(f_{u}+f_{d}+f_{s}-1\right) \approx-0.20$. 
For the second operator, $\mathcal{O}_{2}^{\text {cRayleigh }}$, we need to know the matrix element of $G \widetilde{G}$ inside a nucleon. This matrix element is related to the anomalous divergence of the iso-singlet axial current operator by

$$
\sum_{i=1, \cdots, n_{f}} \partial^{\mu}\left(\bar{\psi}_{i} \gamma_{\mu} \gamma_{5} \psi_{i}\right)=\frac{n_{f}}{4 \pi} \alpha_{s} G_{\mu \nu}^{a} \widetilde{G}^{a \mu \nu}+\sum_{i=1, \cdots, n_{f}} 2 i m_{i} \bar{\psi}_{i} \gamma_{5} \psi_{i}
$$

In the large- $N_{c}$ and chiral limit and using the relation $\left\langle N\left|\bar{u} i \gamma_{5} u+\bar{d} i \gamma_{5} d+\bar{s} i \gamma_{5} s\right| N\right\rangle=0$, one finds [28-30]

$$
\left\langle p\left|\frac{\alpha_{s}}{8 \pi} G_{\mu \nu}^{a} \widetilde{G}^{a \mu \nu}\right| p\right\rangle=389 \mathrm{MeV} \equiv \eta_{p} m_{p}, \quad\left\langle n\left|\frac{\alpha_{s}}{8 \pi} G_{\mu \nu}^{a} \widetilde{G}^{a \mu \nu}\right| n\right\rangle=-2 \mathrm{MeV} \equiv \eta_{n} m_{n},
$$

where the dimensionless parameters are $\eta_{p} \approx 0.41$ and $\eta_{n} \approx-0.0021$ (the instanton calculation in ref. [31] obtained dramatically different numbers). The large difference between $\eta_{p}$ and $\eta_{n}$ indicates a large isospin violation for pseudo-scalar coupling to nucleons. One can then use the matrix elements to translate $\mathcal{O}_{2}$ to the interaction between dark matter and nucleons, giving

$$
\frac{2 i \eta_{N} m_{N}}{\Lambda_{2}^{2}}\left(X X-X^{\dagger} X^{\dagger}\right) \bar{N} i \gamma_{5} N
$$

In the non-relativistic limit, one has $\bar{N} i \gamma_{5} N \approx 2 i \vec{q} \cdot \vec{s}$ with $\vec{q}$ as the exchange momentum of the scattering process and $\vec{s}$ as the spin of a nucleon. So, for this interaction, we have both spin-dependent and momentum-suppressed scattering. The spin-dependent differential scattering cross section is

$$
\frac{d \sigma_{X N}^{\mathrm{SD}}}{d \cos \theta}=\frac{\eta_{N}^{2} m_{N}^{2}}{2 \pi \Lambda_{2}^{4}} \frac{q^{2}}{\left(m_{N}+M_{X}\right)^{2}} .
$$

For $M_{X} \sim 100 \mathrm{GeV}$, a small cutoff scale of $\Lambda_{2} \sim 100 \mathrm{GeV}$, and a typical exchange momentum of $q \sim \mu_{X A} v \sim 100 \mathrm{MeV}$, the spin-dependent scattering cross section of dark matter off a proton is $10^{-7} \mathrm{pb}$. This is far below the current direct detection experimental bound $[32,33]$.

\subsection{Collider constraints}

For the two operators considered here, the universal signature at the LHC is that of a mono-jet plus missing transverse energy [34, 35]. For fermion dark matter coupling to two gluons, both the CMS [36] and ATLAS [37] collaborations have imposed limits on the cutoffs of the effective operators. To estimate the constraints on our scalar dark matter case, we use FeynRules [38] to generate a model file for MadGraph [39]. We then use Pythia [40] to shower and hadronize the parton-level events. Finally, we use PGS [41] to cluster hadrons into jets and simulate detector effects.

Following the same analysis procedure from the CMS collaboration in ref. [36] at $8 \mathrm{TeV}$ and with $19.7 \mathrm{fb}^{-1}$ luminosity, we have found that imposing a cut on the missing transverse energy $E_{T}^{\text {miss }}>500 \mathrm{GeV}$ (or requiring less than 164 signal events) provides the strongest bounds for a wide range of dark matter masses. We show the constraints on the cutoffs of the effective operators in table 1 for different dark matter masses. The constraints stay 


\begin{tabular}{|c|c|c|}
\hline$M_{X}(\mathrm{GeV})$ & $\Lambda_{1}(\mathrm{GeV})$ & $\Lambda_{2}(\mathrm{GeV})$ \\
\hline 1 & 130 & 170 \\
\hline 10 & 120 & 180 \\
\hline 100 & 120 & 180 \\
\hline 200 & 110 & 160 \\
\hline 400 & 90 & 130 \\
\hline
\end{tabular}

Table 1. The collider constraints on the cutoff of the effective operators for different dark matter masses at $90 \% \mathrm{CL}$. The mono-jet analysis with $E_{T}^{\text {miss }}>500 \mathrm{GeV}$ from the CMS collaboration in ref. [36] has been used.

constant for light dark matter below $100 \mathrm{GeV}$ and become weaker as one increases the dark matter mass beyond around $100 \mathrm{GeV}$. One can also see that for heavier dark matter beyond $200 \mathrm{GeV}$, the constraints become much weaker such that the cutoff is even below $M_{X}$, which indicates a breakdown of the perturbative description of the effective field theory.

\section{Simplified UV-completion models}

As already can be seen from table 1, the constraints on the cutoffs of the contact operators from the mono-jet searches are not that stringent. For a $100 \mathrm{GeV}$ dark matter particle, the constrained cutoff is just comparable to the dark matter mass. This calls for UV-completed models to reduce the uncertainties from an effective field theory description. In this section, we consider several classes of models to illustrate that collider signatures beyond the monojet may provide a more sensitive probe of dark matter chromo-Rayleigh interactions.

Introducing only $X$ and its allowed couplings to the SM under the dark $\mathcal{Z}_{2}$ symmetry, the renormalization interactions are

$$
\mathcal{L}_{X} \supset \partial_{\mu} X^{\dagger} \partial^{\mu} X-M_{X}^{\prime 2} X^{\dagger} X-\frac{\lambda_{X}}{4}\left(X^{\dagger} X\right)^{2}-\lambda_{H X} X^{\dagger} X H^{\dagger} H
$$

where $H$ is the SM Higgs doublet and we choose a positive quartic coupling of $\lambda_{X}>0$. After electroweak symmetry breaking, $X$ receives a contribution to its mass from the Higgs mechanism and is assumed to have a positive mass of $M_{X}^{2}=M_{X}^{\prime 2}+v^{2} \lambda_{H X} / 2$, where $v$ is the Higgs vacuum expectation value. Similar to the Higgs-portal dark matter models, the SM Higgs boson can decay into two dark matter particles for $M_{X}<M_{h} / 2$. At $7 \mathrm{TeV}$ and $8 \mathrm{TeV}$ LHC, the searches for the Higgs boson invisible decays have constrained the invisible branching ratio to be less than 0.28 at $95 \%$ C.L. [42, 43], which can be translated into a bound on $\lambda_{H X}$ as $\lambda_{H X} \lesssim 0.015$ for a light dark matter mass.

Given the effective coupling of the Higgs boson with two gluons in the SM, one automatically generates the dark matter-gluon coupling of eq. (2.1). The additional introduction of a color-neutral pseudo-scalar, which appears for instance in two-Higgs-doublet-portal dark matter models, can generate the dark matter chromo-Rayleigh interaction of eq. (2.2) (see e.g. refs. [19, 20, 44, 45]). As the existing search strategies for the Higgs-portal and 

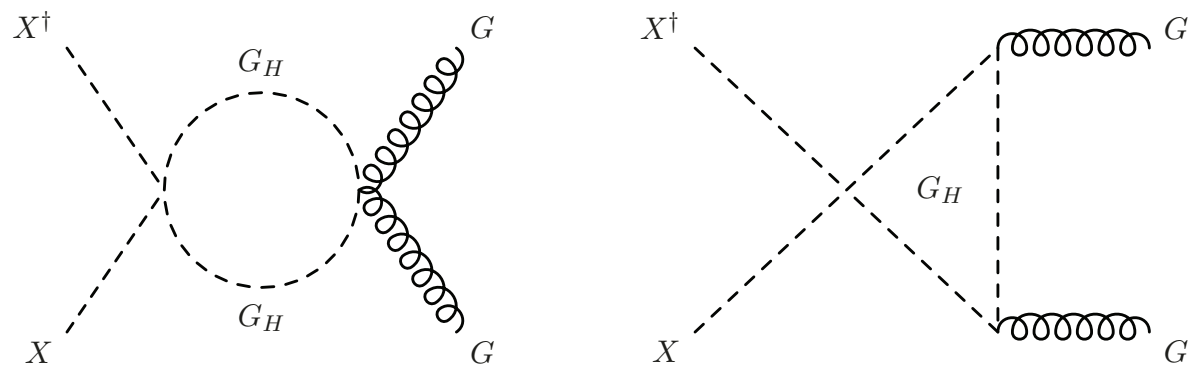

Figure 1. Representative loop diagrams for generating $\mathcal{O}_{1}^{\text {cRayleigh }}$ via colored scalar loops.

pseudo-scalar-portal dark matter cover this class of UV-completions [42], we consider two other ways to UV-complete the chromo-Rayleigh dark matter interactions with more novel collider signatures.

\subsection{QCD-charged particle mediation for $\mathcal{O}_{1}^{\text {cRayleigh }}$}

In this subsection, we examine a class of models with additional QCD-charged scalars to generate the effective chromo-Rayleigh interaction of $\mathcal{O}_{1}^{\text {cRayleigh }}$. For specificity's sake, we introduce a real color-octet and electroweak-singlet scalar, ${ }^{1} G_{H}^{a}$ with $a=1, \cdots, 8$ and a mass of $M_{G_{H}}$, which, for example, appears in the Renormalizable Coloron Model (ReCoM) [46, 47]. The scalar Lagrangian for this theory is

$$
\begin{aligned}
\mathcal{L}_{G_{H}} \supset & \frac{1}{2} D_{\mu} G_{H}^{a} D^{\mu} G_{H}^{a}-\frac{1}{2} M_{G_{H}}^{\prime 2} G_{H}^{a} G_{H}^{a}-\frac{\lambda_{G}}{8}\left(G_{H}^{a} G_{H}^{a}\right)^{2}-\frac{\lambda_{H G}}{2} G_{H}^{a} G_{H}^{a} H^{\dagger} H \\
& -\frac{\lambda_{X G}}{2} G_{H}^{a} G_{H}^{a} X^{\dagger} X+\mathcal{L}_{\text {even(odd) }},
\end{aligned}
$$

where $D_{\mu}$ is the covariant derivative. $\mathcal{L}_{\text {even(odd) }}$ is specific to the identification of $G_{H}$ as even (odd) under the dark $\mathcal{Z}_{2}$ symmetry,

$$
\begin{aligned}
\mathcal{L}_{\text {even }} & =-\mu_{G} d^{a b c} G_{H}^{a} G_{H}^{b} G_{H}^{c}, \\
\mathcal{L}_{\text {odd }} & =-\eta_{X G} d^{a b c} G_{H}^{a} G_{H}^{b} G_{H}^{c}\left(X+X^{\dagger}\right),
\end{aligned}
$$

with $d^{a b c}$ the totally symmetric $\mathrm{SU}(3)_{\mathrm{QCD}}$ tensor. The scalar octet $G_{H}$ also receives a contribution to its mass from the Higgs mechanism, which is $M_{G_{H}}^{2}=M_{G_{H}}^{\prime 2}+v^{2} \lambda_{H G} / 2$. We note that a large value of $\lambda_{H G} / M_{G_{H}}^{2}$ can modify the effective interaction of the Higgs boson coupling to two gluons [48-52]. Using the limits from the LHC Run 1 [53], the limit is $\left|M_{G_{H}} / \sqrt{\lambda_{G H}}\right| \gtrsim 1.9 v$.

At one-loop level, the effective operator can be generated through the diagrams in figure 1 and has the following calculated form

$$
F(\tau) \frac{\lambda_{X G}}{8 M_{G_{H}}^{2}} \frac{\alpha_{s}}{4 \pi} X^{\dagger} X G_{\mu \nu}^{a} G^{a \mu \nu},
$$

where the form factor $F(\tau)$, with $\tau=\left(p_{1}+p_{2}\right)^{2} /\left(4 M_{G_{H}}^{2}\right)$, is one in the limit $\tau \rightarrow 0$. Here, $p_{1}$ and $p_{2}$ are the gluon momenta pointing to the vertex. In the limit that $M_{G_{H}} \gg M_{X}$

\footnotetext{
${ }^{1} \mathrm{~A}$ similar analysis can be performed for other QCD representations.
} 
and with small gluon momenta much below $\left|p_{1}+p_{2}\right|$, one can match the colored particle mass to the effective operator cutoff defined in eq. (2.1) to obtain

$$
\Lambda_{1}^{2}=\frac{8}{\lambda_{X G}} M_{G_{H}}^{2} .
$$

For this class of UV-complete models, one can also work out the collider constraints. Depending on whether the color-octet is odd or even under the dark matter $\mathcal{Z}_{2}$, one has different decay channels for $G_{H}^{a}$. If it is $\mathcal{Z}_{2}$-even, the cubic self-interaction of $\mathcal{L}_{\text {even }}$ can make $G_{H}$ unstable. This introduces the decay of $G_{H}$ into two gluons at one loop with a width of $[46,54]$

$$
\Gamma\left(G_{H} \rightarrow g g\right)=\frac{15 \alpha_{s}^{2} \mu_{G}^{2}}{128 \pi^{3} M_{G_{H}}}\left(\frac{\pi^{2}}{9}-1\right)^{2} .
$$

$\mathcal{Z}_{2}$-even $G_{H}$ can be pair-produced at the LHC from their QCD interactions, which then decay into paired dijets. Following the same reinterpretation of the experimental data as in ref. [55], we find that the current searches for paired dijet resonances at the $8 \mathrm{TeV} \mathrm{LHC}$ have set a constraint on its mass of [56]

$$
M_{G_{H}} \gtrsim 520 \mathrm{GeV}, \quad \text { for } \mathcal{Z}_{2} \text {-even } G_{H},
$$

where we have only included the QCD productions.

If $G_{H}$ is $\mathcal{Z}_{2}$-odd, the dark matter particle $X$ has to appear in the $G_{H}$ decay products. The operators mediating $G_{H}$ decaying to $X$ first occur at the dimension six level and contain the following two parity-conserving operators

$$
\frac{D_{\mu} G_{H}^{a} \partial_{\nu} X G^{a \mu \nu}}{\Lambda_{1}^{\prime 2}}+\text { h.c. }, \quad \frac{G_{H}^{a} X \widetilde{H} \bar{Q}_{L} t^{a} t_{R}}{\Lambda_{1}^{\prime 2}}+h . c .
$$

Here, $t^{a}$ with $a=1,2, \cdots, 8$ are the $\mathrm{SU}(3)_{\mathrm{QCD}}$ generators. For the second operator, we have only included the top quark by assuming that the coupling is proportional to the quark mass. Using the equation of motion, $D_{\mu} G^{a \mu \nu}=-g_{s} \bar{q} \gamma^{\nu} t^{a} q$, the first decay-inducing operator becomes

$$
\frac{g_{s}}{\Lambda_{1}^{\prime 2}} G_{H}^{a} \partial_{\nu} X \bar{q} \gamma^{\nu} t^{a} q
$$

As a result, the decay channels of the colored state $G_{H}$ are mainly $G_{H} \rightarrow X \bar{q} q$. For each flavor, the three-body decay width is calculated to be

$$
\begin{aligned}
& \Gamma\left(G_{H} \rightarrow X \bar{q} q\right)= \\
& \frac{g_{s}^{2}}{3 \cdot 2^{9} \pi^{3} \Lambda_{1}^{\prime}} \int_{4 m_{q}^{2}}^{\left(M_{G_{H}}-M_{X}\right)^{2}} d s\left(1+\frac{2 m_{q}^{2}}{s}\right)\left(1-\frac{4 m_{q}^{2}}{s}\right)^{1 / 2}\left[\frac{M_{X}^{4}+\left(M_{G_{H}}^{2}-s\right)^{2}-2 M_{X}^{2}\left(M_{G_{H}}^{2}+s\right)}{M_{G_{H}}^{2}}\right]^{3 / 2} .
\end{aligned}
$$

Choosing $g_{s}=1.1, M_{G_{H}}=500 \mathrm{GeV}, M_{X}=10 \mathrm{GeV}, \Lambda_{1}^{\prime}=1 \mathrm{TeV}$, and neglecting the quark mass, $\Gamma=0.0002 \mathrm{GeV}$ for each flavor and $G_{H}$ can decay promptly for collider studies.

From the above assumptions, the collider signature for pair-produced $\mathcal{Z}_{2}$-odd $G_{H}$ contains $4 j+E_{T}^{\text {miss }}, t \bar{t}+2 j+E_{T}^{\text {miss }}$ and $2 t+2 \bar{t}+E_{T}^{\text {miss }}$. Generically, the top-quark rich final state can be easily searched for at the LHC. So, we concentrate on the first operator in eq. (3.9) and derivate a more conservative bound based mainly on the $4 j+E_{T}^{\text {miss }}$ final state. Following the analysis in ref. [57] at the $8 \mathrm{TeV} \mathrm{LHC}$ with $19.5 \mathrm{fb}^{-1}$, we find that the set of cuts 
with $300<H_{T}<450 \mathrm{GeV}, 800<H_{T}<1000 \mathrm{GeV}, 3 \leq N_{\text {jets }} \leq 5$ with $p_{T}(j)>50 \mathrm{GeV}$ and $|\eta|<2.5$ provide the best constraint. For $M_{G_{H}}=600 \mathrm{GeV}$ and $M_{X}=10 \mathrm{GeV}$, we have $\operatorname{Br}\left(G_{H} \rightarrow 2 j+X\right)=97.5 \%$ and $\operatorname{Br}\left(G_{H} \rightarrow t \bar{t}+X\right)=2.5 \%$. The production cross section at the $8 \mathrm{TeV}$ LHC after this set of cuts is approximately $1.9 \mathrm{fb} \times K$ with a $K$-factor of 1.8 [58]. This amounts to a total of 65.4 events at $19.5 \mathrm{fb}^{-1}$, which is very close to the allowed number of signal events (65.6 from ref. [57]) at $90 \%$ C.L. So, for a large mass splitting between the color-octet state $G_{H}$ and the dark matter $X$, the current LHC bound is

$$
M_{G_{H}} \gtrsim 600 \mathrm{GeV}, \quad \text { for } \mathcal{Z}_{2} \text {-odd } G_{H} .
$$

Independent of how $G_{H}$ decays, we have set a constraint on the $G_{H}$ mass to be above $500-600 \mathrm{GeV}$. Using the relation in eq. (3.6), this constraint translates to a limit on the effective cutoff scale of $\Lambda_{1} \gtrsim 1.5-1.7 \mathrm{TeV}$ for $\lambda_{X G}=1$, which is far more stringent than the limits in table 1 set from the mono-jet search. To compare with the limits from direct detection experiments, we show the reinterpreted collider limits using $\Lambda_{1}>1.7 \mathrm{TeV}$ for the first chromo-Rayleigh interaction in the left panel of figure 7 . The left panel of figure 7 shows a comparison between the limits on the scattering cross section $\sigma_{X N}^{\mathrm{SI}}$ obtained from direct detection, monojet, and $4 j+E_{T}^{\text {miss }}$ searches.

\subsection{QCD-charged particle mediation for $\mathcal{O}_{2}^{\text {cRayleigh }}$}

To UV-complete the operator $\mathcal{O}_{2}^{\text {cRayleigh }}$, we introduce a pair of vector-like fermions, which appear in a wide range of new physics models like the Kaluza-Klein fermions in the universal extra dimensional model [59] or the top partners in the little Higgs model with $T$-parity [60]. We fix the quantum numbers of these fermions to be the same as $u_{R}$ for later convenience. The Lagrangian for this sector is then

$$
\begin{aligned}
\mathcal{L}_{\psi} \supset & \bar{\psi}_{1}\left(i \not D-m_{\psi_{1}}\right) \psi_{1}+\bar{\psi}_{2}\left(i \not D-m_{\psi_{2}}\right) \psi_{2}-\left(\lambda_{\psi_{1(2)}} \bar{Q}_{L} \widetilde{H} \psi_{1(2)}+\text { h.c. }\right) \\
& -y_{1}\left(X+X^{\dagger}\right)\left(\bar{\psi}_{1} \psi_{2}+\bar{\psi}_{2} \psi_{1}\right)-y_{2}\left(X-X^{\dagger}\right)\left(\bar{\psi}_{1} \gamma_{5} \psi_{2}+\bar{\psi}_{2} \gamma_{5} \psi_{1}\right)
\end{aligned}
$$

To conserve the dark $\mathcal{Z}_{2}$ symmetry, one of the fermion triplets must be $\mathcal{Z}_{2}$-odd while the other is $\mathcal{Z}_{2}$-even. Only the $\mathcal{Z}_{2}$-even fermion has an allowed Yukawa coupling with the standard model Higgs boson. Using the freedom of field redefinitions of $\psi_{1}$ and $\psi_{2}$, one can keep the first coupling, $y_{1}$, to be a real number while the second coupling, $y_{2}$, may in general be complex. We choose $y_{2}$ to be a real number to satisfy $C P$ symmetry. In terms of the components, $X=\left(X_{R}+i X_{I}\right) / \sqrt{2}$, we have the interactions

$$
\mathcal{L} \supset-\sqrt{2} y_{1} X_{R}\left(\bar{\psi}_{1} \psi_{2}+\bar{\psi}_{2} \psi_{1}\right)-\sqrt{2} i X_{I}\left(y_{2} \bar{\psi}_{1} \gamma_{5} \psi_{2}+y_{2} \bar{\psi}_{2} \gamma_{5} \psi_{1}\right)
$$

To preserve $C$ and $P$, one has $X_{R}$ to be $C$-even and $P$-even, and $X_{I}$ to be $C$-even and $P$-odd. In terms of $X_{R}$ and $X_{I}$, we have the effective operator

$$
\mathcal{O}_{2}^{\text {cRayleigh }}=-\frac{\alpha_{s}}{2 \pi \Lambda_{2}^{2}} X_{R} X_{I} G_{\mu \nu}^{a} \widetilde{G}^{a \mu \nu}
$$

which conserves both $C$ and $P$. 

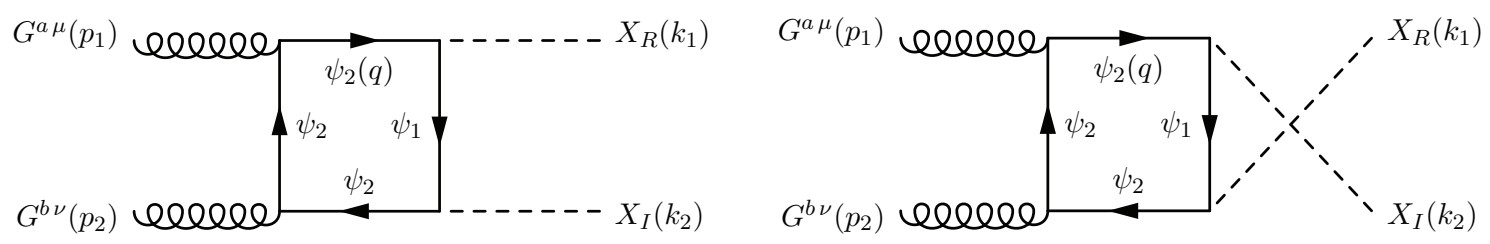

Figure 2. Representative loop diagrams for generating $\mathcal{O}_{2}^{\text {cRayleigh }}$ via colored fermion loop.

At one-loop level, one has the box diagrams in figure 2 to generate the effective operator $\mathcal{O}_{2}^{\text {cRayleigh }}$. In the heavy particle limit with $m_{\psi_{1}}, m_{\psi_{2}} \gg M_{X_{R}}, M_{X_{I}}$, we have the matching condition (see appendix A for a more detailed calculation)

$$
\Lambda_{2}^{2}=\frac{2 m_{\psi_{1}} m_{\psi_{2}}}{\left|y_{1} y_{2}\right|}
$$

For this specific UV-completion model, the first chromo-Rayleigh interaction can also be generated, since it does not break any discrete symmetries.

If $\operatorname{Im}\left(y_{1} y_{2}\right) \neq 0$, the Lagrangian in eq. (3.14) is $P$-conserving but $C$-breaking, so $C P$ is also broken. ${ }^{2}$ The lowest dimensional effective operator that is $C$-odd and $P$ even is at dimension-10, for instance $X_{R} X_{I} d_{a b c}\left(D_{\mu} G_{\alpha \beta}^{a}\right)\left(D_{\nu} G^{b \nu \alpha}\right) \widetilde{G}^{c \beta \mu}$, further suppressed by powers of the cutoff scale. If parity was broken, lower-dimensional operators like $X_{R} X_{I} G_{\mu \nu}^{a} G^{a \mu \nu}$ could be generated with a stringent bound from the neutron electric dipole moment.

As mentioned before, in order to conserve the dark $\mathcal{Z}_{2}$ symmetry the two fields, $\psi_{1}$ and $\psi_{2}$, should have opposite dark parity. Fixing the mass relation $m_{\psi_{2}}>m_{\psi_{1}}$, we have two cases. The case $\mathrm{A}$ has $\mathcal{Z}_{2}$-even $\psi_{1}$ and $\mathcal{Z}_{2}$-odd $\psi_{2}$ and the case $\mathrm{B}$ has $\mathcal{Z}_{2}$-even $\psi_{2}$ and $\mathcal{Z}_{2}$-odd $\psi_{1}$. For both cases, we introduce the following dimension-5 operator to mediate the $\mathcal{Z}_{2}$-even particle decaying into two jets,

$$
\frac{g_{s}^{2}}{4 \pi \Lambda_{2}^{\prime}} \bar{\psi}_{i L} \sigma^{\mu \nu} t^{a} u_{R} \widetilde{G}_{a}^{\mu \nu}
$$

Here, $i=1(2)$ for case $\mathrm{A}(\mathrm{B})$. As a result of this decay mode, the unstable particle $\psi_{i}$ behaves as a dijet resonance at colliders. One could also consider other potential decay channels by introducing the electroweak dipole moment operator, which has a clearer signature at colliders. The Yukawa operator with a coefficient, $\lambda_{\psi_{i}}$, in eq. (3.13) can also mediate the $\mathcal{Z}_{2}$-even $\psi_{i}$ decaying to one up quark plus $Z$ or $h$. The constraint on the mass of $\mathcal{Z}_{2}$-even $\psi_{i}$ is above between $720 \mathrm{GeV}$ and $920 \mathrm{GeV}$ for different branching fractions [62]. In our study, we will assume a negligible value of $\lambda_{\psi_{i}}$ and concentrate on the more challenging fully-hadronic channel.

For case $\mathrm{A}$ with a $\mathcal{Z}_{2}$-even $\psi_{1}$, the collider signature of $\psi_{1}$ is pair-produced dijet resonances. The searches at the $8 \mathrm{TeV}$ LHC have set a constraint on its mass [56] as

\footnotetext{
${ }^{2}$ For a complex $y_{2}$ with $C P$-violating interactions, one may wonder about generating the Weinberg operator [61], $f_{a b c} G_{\mu \rho}^{a} G_{\nu}^{b \rho} \widetilde{G}^{c \mu \nu}$. We note that the $P$ conservation forbids the generation of the effective Weinberg operator.
} 

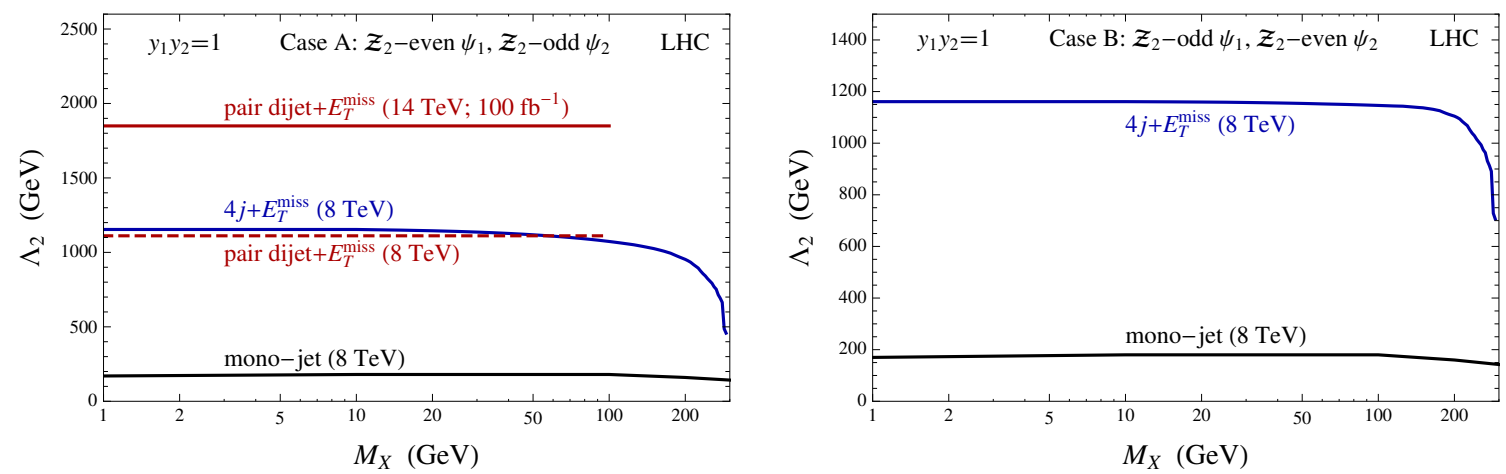

Figure 3. Left panel: constraints on the cutoff of the effective operator $\mathcal{O}_{2}^{\text {cRayleigh }}$ for case A with $\mathcal{Z}_{2}$-even $\psi_{1}$ and $\mathcal{Z}_{2}$-odd $\psi_{2}$. Right panel: constraints for case B with $\mathcal{Z}_{2}$-odd $\psi_{1}$ and $\mathcal{Z}_{2}$-even $\psi_{2}$.

$m_{\psi_{1}} \gtrsim 500 \mathrm{GeV}$. Although the signature is interesting by itself, the discovery of this dijet resonance can not prove that dark matter has been produced at colliders. For the $\mathcal{Z}_{2}$-odd $\psi_{2}$ particle, ithe decay channel is $\psi_{2} \rightarrow X+\psi_{1} \rightarrow X+2 j$. Depending on the mass splitting of $\psi_{1}$ and $\psi_{2}$, one has $\psi_{1}$ to be off-shell for $m_{\psi_{2}}-m_{\psi_{1}}<M_{X}$ and on-shell for $m_{\psi_{2}}-m_{\psi_{1}}>M_{X}$. For the off-shell intermediate $\psi_{1}$ case, this signature is very similar to the SUSY squark searches with a heavy gluino [57], except with a larger production cross section than a single flavor squark. For dark matter mass $M_{X} \lesssim 100 \mathrm{GeV}$, the constraint on the $\psi_{2}$ mass is $m_{\psi_{2}} \gtrsim 850 \mathrm{GeV}$, assuming that the signal acceptance is similar to the squark one [57]. For a heavy dark matter mass close to $290 \mathrm{GeV}$, the constraint becomes weaker and is $m_{\psi_{2}} \gtrsim 500 \mathrm{GeV}$. Using the conversion formula in eq. (3.16), we show the constraints from $4 j+E_{T}^{\text {miss }}$ on the effective cutoff for different dark matter masses in the left panel of figure 3 , by setting $m_{\psi_{1}}=m_{\psi_{2}}-M_{X}$ and $y_{1} y_{2}=1$.

For the on-shell $\psi_{1}$ case, the signature is more interesting with a pair of dijet resonances plus a large $E_{T}^{\text {miss }}$. The relevant production Feynman diagram is shown in the left panel of figure 4. We perform a detailed collider study for this interesting signature in section 3.3. As a comparison, in the left panel of figure 3 we show the interpreted constrains for $M_{X} \lesssim 100 \mathrm{GeV}$ on the matched cutoff from eq. (3.16) for both the $8 \mathrm{TeV}$ LHC with $19.5 \mathrm{fb}^{-1}$ and the $14 \mathrm{TeV}$ LHC with $100 \mathrm{fb}^{-1}$. One can see a clear improvement on constraining this effective chromo-Rayleigh interaction from a dedicated search beyond the simple mono-jet signature.

For case $\mathrm{B}$ with $\mathcal{Z}_{2}$-odd $\psi_{1}$ and $\mathcal{Z}_{2}$-even $\psi_{2}$, the searches for pair-produced dijet resonances provide a constraint of $m_{\psi_{2}} \gtrsim 500 \mathrm{GeV}$. The decay of $\psi_{1}$ needs to go through an off-shell $\psi_{2}$ via $\psi_{1} \rightarrow X+\psi_{2}^{*} \rightarrow X+2 j$. Applying the SUSY squark searches with a heavy gluino [57], the constraint on the $\psi_{1}$ mass is $m_{\psi_{2}} \geq m_{\psi_{1}} \gtrsim 850 \mathrm{GeV}$ for $M_{X} \lesssim 100 \mathrm{GeV}$ and $m_{\psi_{1}} \gtrsim 500 \mathrm{GeV}$ for $M_{X}$ close to $290 \mathrm{GeV}$. In the right panel of figure 3, we show the reinterpreted constraints on the effective cutoff for $y_{1} y_{2}=1$. 

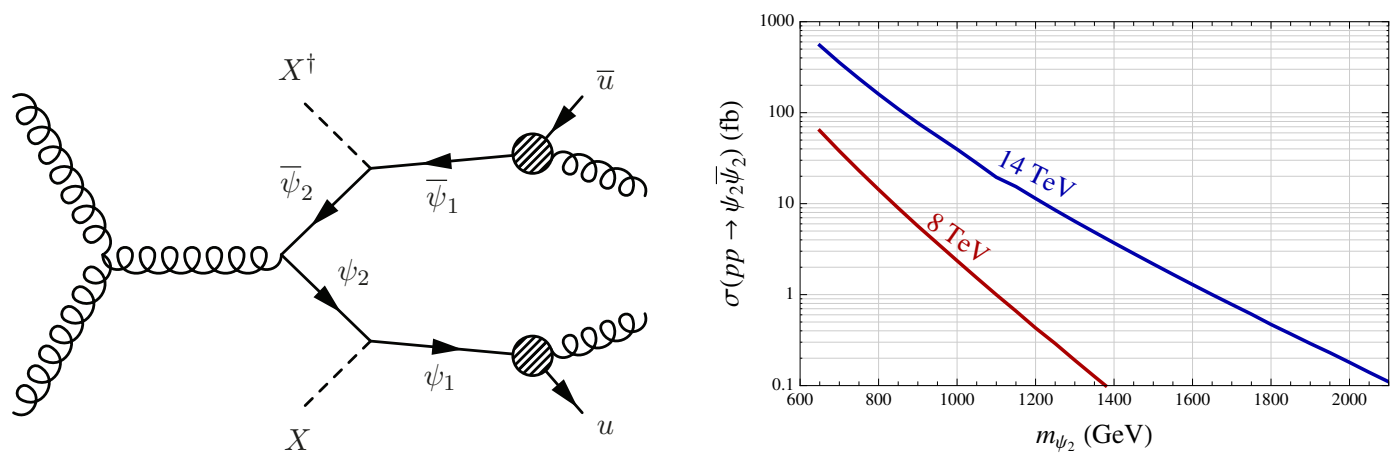

Figure 4. Left panel: the representive Feynman diagram for the process of $p p \rightarrow \psi_{2} \bar{\psi}_{2} \rightarrow X+$ $\psi_{1}+X^{\dagger}+\bar{\psi}_{1} \rightarrow X X^{\dagger}+4 j$. Right panel: the tree-level production cross sections of $p p \rightarrow \psi_{2} \bar{\psi}_{2}$.

\subsection{Pair-produced dijet resonances plus $E_{T}^{\text {miss }}$}

In this section, we perform a detailed collider study for the process of $p p \rightarrow \psi_{2} \bar{\psi}_{2} \rightarrow$ $X+\psi_{1}+X^{\dagger}+\bar{\psi}_{1} \rightarrow X X^{\dagger}+4 j$. The collider signature is pair-produced dijet resonances plus missing transverse energy. In the left panel of figure 4, we show the Feynman diagram for this process. The QCD production cross sections for $\psi_{2} \bar{\psi}_{2}$ at the LHC are the same as vector-like $t^{\prime} \bar{t}^{\prime}$ [63]. We show the tree-level production cross sections, calculated using MadGraph [39], in the right panel of figure 4. In the following analysis, we will ignore the signal $K$-factor because we will use tree-level cross sections for backgrounds. ${ }^{3}$

The main SM background comes from $Z / W^{ \pm}+n$ jets with $Z \rightarrow \nu \bar{\nu}$ and leptonic decays of $W^{ \pm}$. After comparing the results from matching parton showers and matrix elements [64], we have found that the background of parton-level $Z / W^{ \pm}+4$ jets with $p_{T}(j)>120 \mathrm{GeV}$ provides a good estimation of total $Z / W^{ \pm}+n$ jets background for the $8 \mathrm{TeV}$ LHC. Therefore, we use $Z / W^{ \pm}+4$ jets as an approximation to save simulation time. There also exist additional, sub-dominant semi-leptonic $t \bar{t}$ backgrounds, which will be kept in our analysis.

Starting from the $8 \mathrm{TeV}$ LHC with $19.5 \mathrm{fb}^{-1}$, we choose the model point $\left(m_{\psi_{1}}, m_{\psi_{2}}\right)=$ $(600,900) \mathrm{GeV}$ as a benchmark to optimize our cuts on kinematic variables. We choose the basic cuts on the jet and missing transverse momenta to be $p_{T}\left(j_{i}\right)>140 \mathrm{GeV}$ and $E_{T}^{\text {miss }}>275 \mathrm{GeV}$ and required at least four jets satisfying the jet $p_{T}$ cut in the final state. To reduce the $t \bar{t}$ and $W^{ \pm}+$jets backgrounds, we also veto events containing a lepton with $p_{T}(\ell)>20 \mathrm{GeV}$. Since our signal has a pair of dijet resonances with the same mass, we choose the combination among three possible dijet pairs with the smallest dijet invariant mass difference, $\Delta m_{\text {dijet }}$. To further reduce the SM background, we show the event fraction histogram distribution in terms of the variable $\Delta m_{\text {dijet }} / m_{\text {avg }}$ in the left panel of figure 5 . Signal events prefer a smaller value of dijet invariant mass difference than background events. As a result, we impose a cut on this variable with $\Delta m_{\text {dijet }} / m_{\text {avg }}<0.4$ to further increase the discovery sensitivity. In the right panel of figure 5 , we show the averaged dijet invariant mass distribution for the signal and backgrounds. Since the signal events

\footnotetext{
${ }^{3}$ One might expect the overall significance in the later analysis to be increased by a factor of $\sim 1.2-1.3$ due to the inclusion of NLO effects.
} 

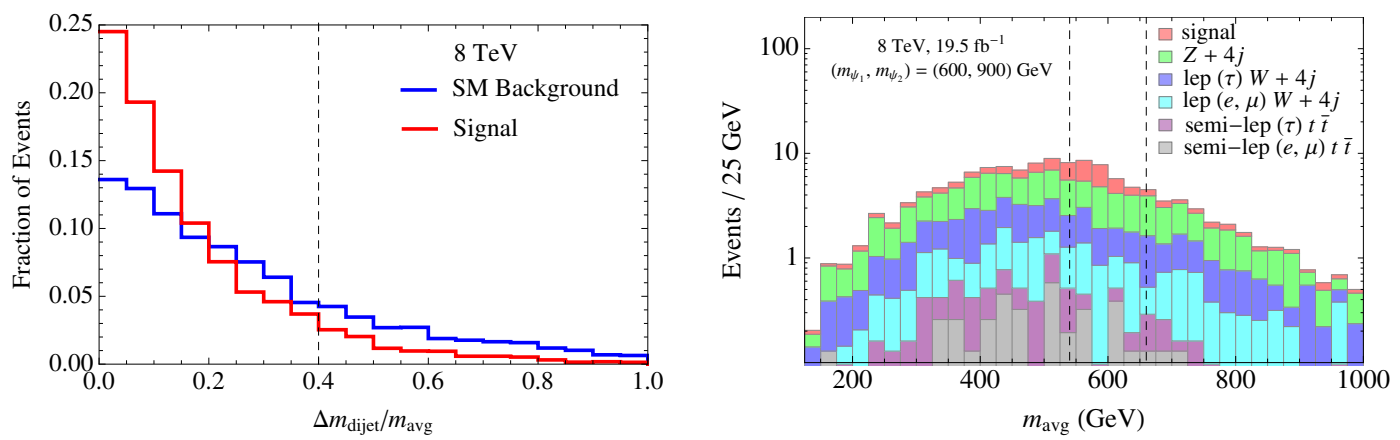

Figure 5. Left panel: the distributions of the fraction of events as a function of $\Delta m_{\text {dijet }} / m_{\text {avg }}$ for the signal of $\left(m_{\psi_{1}}, m_{\psi_{2}}\right)=(600,900) \mathrm{GeV}$ and the summed background. Right panel: the averaged dijet invariant mass distributions for the signal and various backgrounds, after the cut of $\Delta m_{\text {dijet }} / m_{\text {avg }}<0.4$
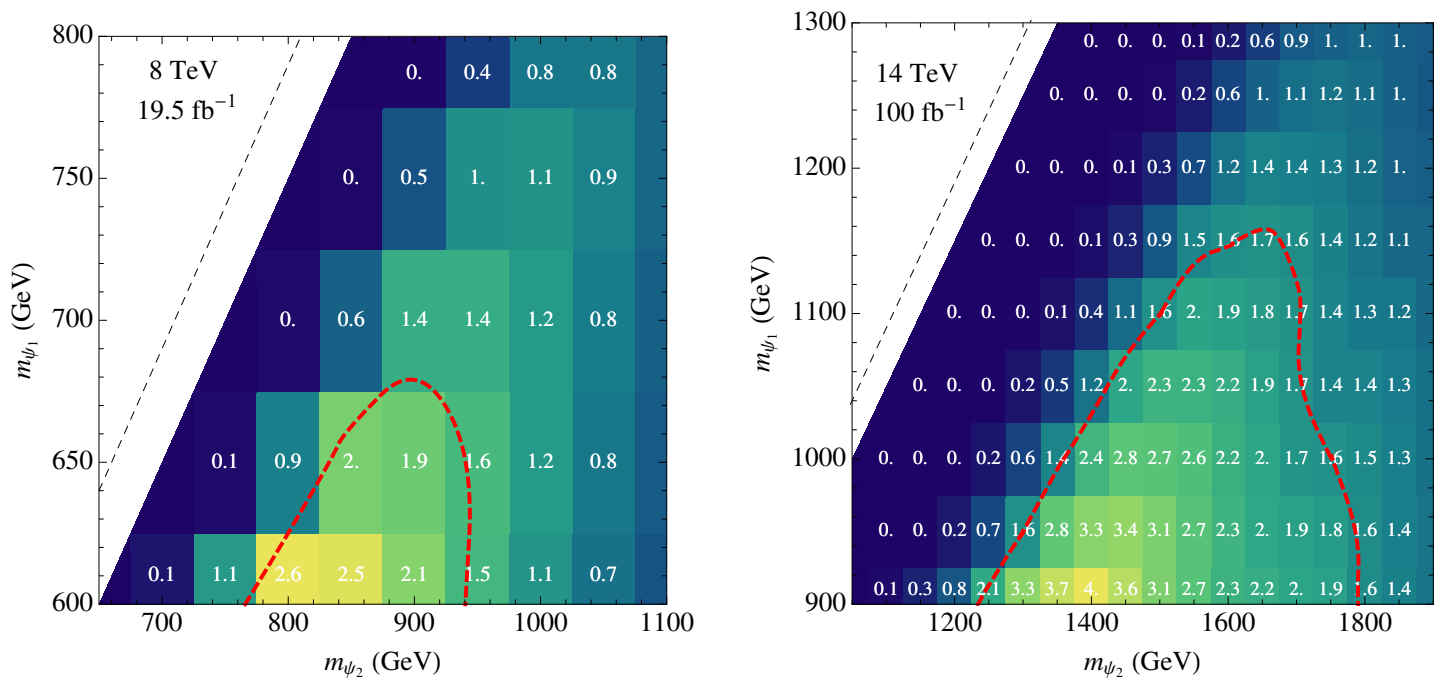

Figure 6. Left panel: the signal significance $S / \sqrt{B}$ for different model points with the cuts optimized for $\left(m_{\psi_{1}}, m_{\psi_{2}}\right)=(600,900) \mathrm{GeV}$ and $M_{X}=10 \mathrm{GeV}$ at the $8 \mathrm{TeV} \mathrm{LHC}$ with $19.5 \mathrm{fb}^{-1}$. The red dashed line is the extrapolated $90 \%$ C.L. exclusion curve. The black dashed line has $m_{\psi_{2}}=m_{\psi_{1}}+M_{X}$. Right panel: the same as the left panel but for $14 \mathrm{TeV}$ with $100 \mathrm{fb}^{-1}$ and the set of cuts for the combined benchmark points $\left(m_{\psi_{1}}, m_{\psi_{2}}\right)=(900,1800) \mathrm{GeV}$ and $(900,1500) \mathrm{GeV}$ with $M_{X}=10 \mathrm{GeV}$.

mainly distribute around the $\psi_{1}$ particle mass, we also impose one additional cut with $\left|m_{\text {avg }}-m_{\psi_{1}}\right|<0.1 m_{\psi_{1}}$ to further improve the sensitivity. With the above cuts, we show the values of $S / \sqrt{B}$ for different mass points in the left panel of figure 6. At $90 \%$ C.L., our simulated results show that the model point of $\left(m_{\psi_{1}}, m_{\psi_{2}}\right)=(650,950) \mathrm{GeV}$ can be covered, which corresponds to $\Lambda_{2} \gtrsim 1.1 \mathrm{TeV}$. Once again we see a dramatic improvement relative to the standard mono-jet signature.

At the $14 \mathrm{TeV}$ LHC with $100 \mathrm{fb}^{-1}$, we choose two benchmark points with $M_{X}=$ $10 \mathrm{GeV}$ and $\left(m_{\psi_{1}}, m_{\psi_{2}}\right)=(900,1800)[(900,1500)] \mathrm{GeV}$. To optimize the discovery sensitivity, we choose the following set of cuts: at least four jets with $p_{T}(j)>200 \mathrm{GeV}$; 
$E_{T}^{\text {miss }}>900[600] \mathrm{GeV} ; \Delta m_{\text {dijet }} / m_{\text {avg }}<0.4[0.3] ;\left|m_{\text {avg }}-m_{\psi_{1}}\right|<0.1 m_{\psi_{1}}$. The signal significances, $S / \sqrt{B}$, for different model points are shown in the right panel of figure 6 , taking the larger $S / \sqrt{B}$ value from between the two benchmark points. We show the combined 90\% C.L. exclusion contour in the red dashed line, which has the lower right region covered by the set of cuts for $(900,1800) \mathrm{GeV}$ and the upper right region by $(900,1500) \mathrm{GeV}$. At $90 \%$ C.L., one can see that the model point of $\left(m_{\psi_{1}}, m_{\psi_{2}}\right)=(950,1800) \mathrm{GeV}$ can be excluded, which corresponds to $\Lambda_{2} \gtrsim 1.8 \mathrm{TeV}$.

\section{Comparison to the direct detection limits}

To compare with the constraints from direct detection experiments, we show the interpreted dark matter-nucleon scattering cross sections from the LHC mono-jet, $4 j+E_{T}^{\text {miss }}$ and paired dijet $+E_{T}^{\text {miss }}$ searches in figure 7 . In the left panel, we show the spin-independent scattering cross section including the constraints from the LUX collaboration [36]. Compared to the direct detection limits, the constraints from the LHC are not strong for heavier dark matter masses, but are more stringent for a light dark matter mass below around $5 \mathrm{GeV}$. Compared to the limits from mono-jet searches, the $4 j+E_{T}^{\text {miss }}$ signature is definitely a more sensitive channel for constraining the spin-independent scattering cross section from the first chromo-Rayleigh interaction, $X^{\dagger} X G_{\mu \nu}^{a} G^{a \mu \nu}$.

In the right panel of figure 7, we show the interpreted collider constrains for the second operator, $i\left(X X-X^{\dagger} X^{\dagger}\right) G_{\mu \nu}^{a} \widetilde{G}^{a \mu \nu}$. Here, we only show the dark matter-proton scattering cross section, since it has a larger value than dark matter-neutron one for the same dark matter mass. For the interpreted limits from collider searches and because of the additional momentum suppression with $q \approx \mu_{X} A v$, we fix the dark matter velocity to be $v=10^{-3}$ and choose a typical target nucleus mass $m_{A}=100 \mathrm{GeV}$ in eq. (2.11). Compared to the constraints from direct detection experiments, the collider searches obviously provide a more sensitive probe of this type of dark matter chromo-Rayleigh interactions.

\section{Discussion and conclusions}

One advantage of introducing simplified models is that the relevant model is complete from the renormalizability point of view. There is no worry about trusting theoretical descriptions of the collider limits like in the effective operator approach. On the other hand, the simplified models typically contain more model parameters and make the comparison with the results from dark matter direct or indirect detection less model-independent. For the simplified models studied here, the collider signatures highly depend on how the QCD charged particles decay. For the first operator, the color-octet scalar can decay into two gluons or two quarks plus the dark matter particle. For the second operator, the two color-triplet fermions could have many other possible decay channels beyond the ones in our paper. For instance, one could replace the operator in eq. (3.17) by $\bar{\psi}_{i L} \sigma^{\mu \nu} u_{R} \widetilde{B}^{\mu \nu}$, which can lead to the decay of $\psi \rightarrow u+\gamma / Z$. The final collider signature could be $2 j+2 \gamma(Z)+E_{T}^{\text {miss }}$ with or without a pair of $j+\gamma(Z)$ resonances. To cover the majority 

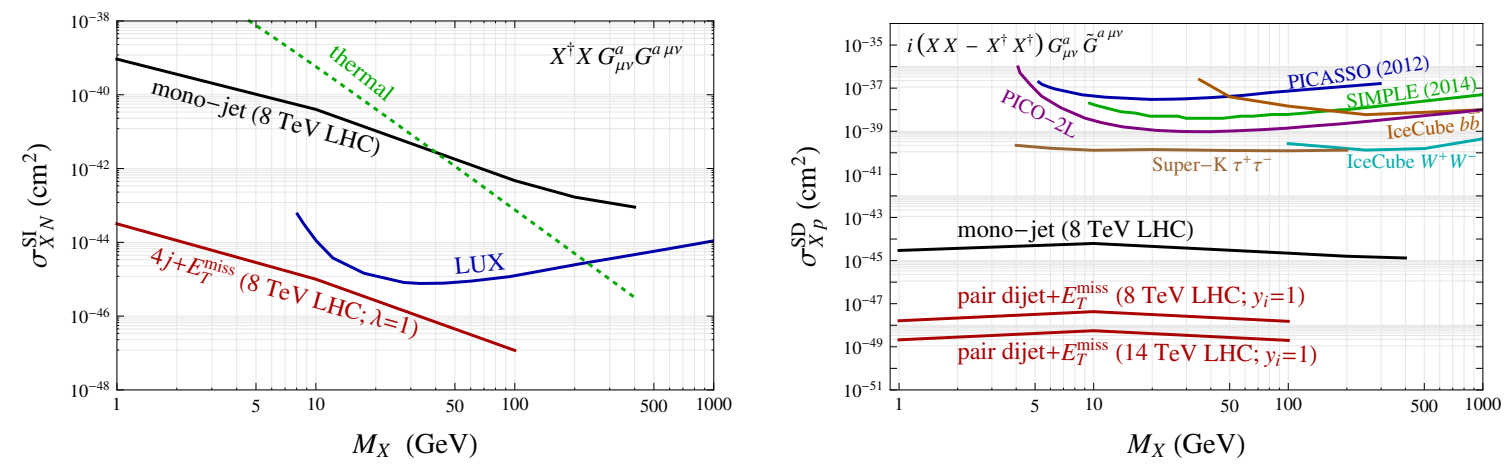

Figure 7. Left panel: the constraints on the dark matter spin-independent scattering cross sections from the LHC mono-jet [36] and multi-jet $+E_{T}^{\text {miss }}$ [57] searches at the $8 \mathrm{TeV}$ LHC with $\sim 20 \mathrm{fb}^{-1}$ and the LUX direct detection experiment [65]. Right panel: the same as the left one but for spindependent dark matter-proton scattering cross sections from the LHC, SIMPLE [66], PICASSO [67], PICO-2L [68], IceCube [69] and Super-K [70]. The scattering cross section is suppressed by both the spin-dependent scattering and the exchanging momenta.

of simplified models for dark matter chromo-Rayleigh interactions, one should search for a wide range of potential signatures.

In conclusion, we have studied simplified models to UV-complete the chromo-Rayleigh interactions of dark matter. For the first operator, a new color-octet scalar particle could be in reach of the LHC. It may decay into just two jets or two jets plus missing transverse energy. The $8 \mathrm{TeV}$ LHC can already constrain this color-octet scalar mass to be above 500$600 \mathrm{GeV}$, which can be translated into a constraint on the cutoff of the effective operator to be above 1.5-1.7 TeV. For the second operator, two QCD-charged fermions are predicted for the simplified model in this paper. A collider study of pair-produced dijet resonances plus missing energy at the $14 \mathrm{TeV}$ LHC can constrain the geometric mean of the two fermion masses above around $800 \mathrm{GeV}$, which can be interpreted as a bound on the effective operator cutoff above around $1.8 \mathrm{TeV}$.

Note added. We note here that during the completion of our paper, another paper [71] appeared containing some overlap with our UV-completion of the first operator, $X^{\dagger} X G_{\mu \nu}^{a} G^{a \mu \nu}$.

\section{Acknowledgments}

We would like to thank Ran Lu for useful discussion. This work is supported by the U. S. Department of Energy under the contract DE-FG-02-95ER40896.

\section{A Loop level calculations for the coefficient of $\mathcal{O}_{2}^{\text {cRayleigh }}$}

Starting from the Lagrangian in eq. (3.14), we match the coefficient for $\mathcal{O}_{2}^{\text {cRayleigh }}$ at oneloop level. Other than the two Feynman diagrams in figure 2, we also have another four diagrams in figure 8 and figure 9 . 

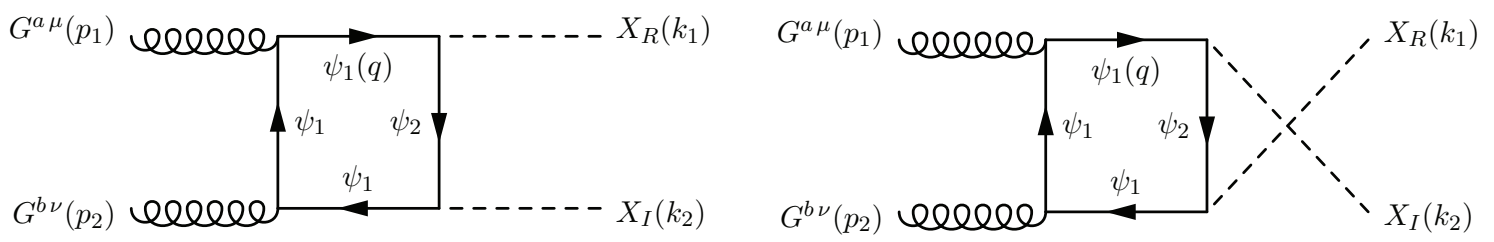

Figure 8. Loop diagrams for generating $\mathcal{O}_{2}^{\text {cRayleigh }}$ via a colored particle in loop.
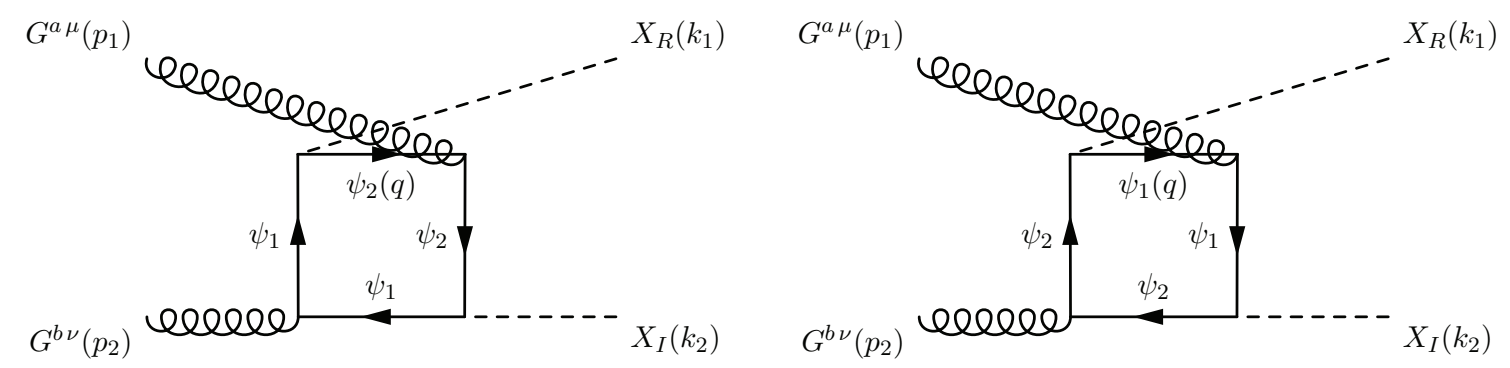

Figure 9. Loop diagrams for generating $\mathcal{O}_{2}^{\text {cRayleigh }}$ via a colored particle in loop.

Summing the two diagrams in figure 2, we have the matrix element as

$$
\begin{aligned}
i \mathcal{M}_{1}= & 4 i g_{s}^{2} y_{1} y_{2} \operatorname{Tr}\left[t^{a} t^{b}\right] \varepsilon_{\mu}\left(p_{1}\right) \varepsilon_{\nu}\left(p_{2}\right) \\
& \times \int \frac{d^{4} q}{(2 \pi)^{4}} \frac{\operatorname{Tr}\left[\left(\not 1+m_{2}\right) \gamma^{\mu}\left(\not q-\not p_{1}+m_{2}\right) \gamma^{\nu}\left(\not q-\not k_{1}-\not k_{2}+m_{2}\right) \gamma^{5}\left(\not q-\not k_{1}+m_{1}\right)\right]}{\left[q^{2}-m_{2}^{2}\right]\left[\left(q-p_{1}\right)^{2}-m_{2}^{2}\right]\left[\left(q-k_{1}-k_{2}\right)^{2}-m_{2}^{2}\right]\left[\left(q-k_{1}\right)^{2}-m_{1}^{2}\right]}
\end{aligned}
$$

In the heavy fermion limit, we have a simplified version of the matrix element as

$$
i \mathcal{M}_{1}=i \frac{g_{s}^{2} y_{1} y_{2}}{4 \pi^{2}} \delta^{a b} \varepsilon_{\mu}\left(p_{1}\right) \varepsilon_{\nu}\left(p_{2}\right) \epsilon^{\mu \nu \rho \sigma} p_{1 \rho} p_{2 \sigma}\left[\frac{-m_{1}^{5}+2 m_{1}^{3} m_{2}^{2} \log \left(m_{1}^{2} / m_{2}^{2}\right)+m_{1} m_{2}^{4}}{m_{2}\left(m_{1}^{2}-m_{2}^{2}\right)^{3}}\right] \text {. }
$$

The two diagrams in figure 8 have a similar answer as $i \mathcal{M}_{2}=i \mathcal{M}_{1}\left(m_{1} \leftrightarrow m_{2}\right)$. The two digrams in figure 9 has a summed matrix as

$$
i \mathcal{M}_{3}=i \frac{g_{s}^{2} y_{1} y_{2}}{2 \pi^{2}} \delta^{a b} \varepsilon_{\mu}\left(p_{1}\right) \varepsilon_{\nu}\left(p_{2}\right) \epsilon^{\mu \nu \rho \sigma} p_{1 \rho} p_{2 \sigma}\left\{\frac{m_{1} m_{2}\left[2\left(m_{1}^{2}-m_{2}^{2}\right)-\left(m_{1}^{2}+m_{2}^{2}\right) \log \left(m_{1}^{2} / m_{2}^{2}\right)\right]}{\left(m_{1}^{2}-m_{2}^{2}\right)^{3}}\right\} .
$$

Add all diagrams together, we have the summed matrix element

$$
i \mathcal{M}_{1}+i \mathcal{M}_{2}+i \mathcal{M}_{3}=\frac{g_{s}^{2} y_{1} y_{2}}{4 \pi^{2} m_{1} m_{2}} \delta^{a b} \varepsilon_{\mu}\left(p_{1}\right) \varepsilon_{\nu}\left(p_{2}\right) \epsilon^{\mu \nu \rho \sigma} p_{1 \rho} p_{2 \sigma}
$$

To match to the coefficient of $\mathcal{O}_{2}^{\text {cRayleigh }}$, one has

$$
\frac{\alpha_{s} y_{1} y_{2}}{\pi m_{1} m_{2}}=\frac{2 \alpha_{s}}{\pi \Lambda_{2}^{2}}
$$

Open Access. This article is distributed under the terms of the Creative Commons Attribution License (CC-BY 4.0), which permits any use, distribution and reproduction in any medium, provided the original author(s) and source are credited. 


\section{References}

[1] M. Cahill-Rowley et al., Complementarity of dark matter searches in the phenomenological MSSM, Phys. Rev. D 91 (2015) 055011 [arXiv:1405.6716] [INSPIRE].

[2] A.L. Erickcek, P.J. Steinhardt, D. McCammon and P.C. McGuire, Constraints on the interactions between dark matter and baryons from the X-ray quantum calorimetry experiment, Phys. Rev. D 76 (2007) 042007 [arXiv:0704.0794] [INSPIRE].

[3] B.A. Dobrescu, D. Hooper, K. Kong and R. Mahbubani, Spinless photon dark matter from two universal extra dimensions, JCAP 10 (2007) 012 [arXiv:0706.3409] [INSPIRE].

[4] J. Bagnasco, M. Dine and S.D. Thomas, Detecting technibaryon dark matter, Phys. Lett. B 320 (1994) 99 [hep-ph/9310290] [INSPIRE].

[5] M.T. Frandsen, U. Haisch, F. Kahlhoefer, P. Mertsch and K. Schmidt-Hoberg, Loop-induced dark matter direct detection signals from gamma-ray lines, JCAP 10 (2012) 033 [arXiv: 1207.3971] [INSPIRE].

[6] J. Liu, B. Shuve, N. Weiner and I. Yavin, Looking for new charged states at the LHC: signatures of magnetic and Rayleigh dark matter, JHEP 07 (2013) 144 [arXiv:1303.4404] [INSPIRE].

[7] S. Chang, R. Edezhath, J. Hutchinson and M. Luty, Effective WIMPs, Phys. Rev. D 89 (2014) 015011 [arXiv:1307.8120] [INSPIRE].

[8] H. An, L.-T. Wang and H. Zhang, Dark matter with t-channel mediator: a simple step beyond contact interaction, Phys. Rev. D 89 (2014) 115014 [arXiv:1308.0592] [INSPIRE].

[9] Y. Bai and J. Berger, Fermion portal dark matter, JHEP 11 (2013) 171 [arXiv:1308.0612] [INSPIRE].

[10] A. DiFranzo, K.I. Nagao, A. Rajaraman and T.M.P. Tait, Simplified models for dark matter interacting with quarks, JHEP 11 (2013) 014 [Erratum ibid. 01 (2014) 162] [arXiv: 1308.2679] [INSPIRE].

[11] O. Buchmueller, M.J. Dolan and C. McCabe, Beyond effective field theory for dark matter searches at the LHC, JHEP 01 (2014) 025 [arXiv: 1308.6799] [INSPIRE].

[12] B. Batell, T. Lin and L.-T. Wang, Flavored dark matter and R-parity violation, JHEP 01 (2014) 075 [arXiv: 1309.4462] [INSPIRE].

[13] M. Papucci, A. Vichi and K.M. Zurek, Monojet versus the rest of the world I: $t$-channel models, JHEP 11 (2014) 024 [arXiv:1402.2285] [INSPIRE].

[14] Y. Bai and J. Berger, Lepton portal dark matter, JHEP 08 (2014) 153 [arXiv:1402.6696] [INSPIRE].

[15] S. Chang, R. Edezhath, J. Hutchinson and M. Luty, Leptophilic effective WIMPs, Phys. Rev. D 90 (2014) 015011 [arXiv:1402.7358] [INSPIRE].

[16] P. Agrawal, Z. Chacko and C.B. Verhaaren, Leptophilic dark matter and the anomalous magnetic moment of the muon, JHEP 08 (2014) 147 [arXiv:1402.7369] [INSPIRE].

[17] M. Garny, A. Ibarra, S. Rydbeck and S. Vogl, Majorana dark matter with a coloured mediator: collider vs direct and indirect searches, JHEP 06 (2014) 169 [arXiv:1403.4634] [INSPIRE]. 
[18] J.-H. Yu, Vector fermion-portal dark matter: direct detection and galactic center gamma-ray excess, Phys. Rev. D 90 (2014) 095010 [arXiv: 1409.3227] [inSPIRE].

[19] M.R. Buckley, D. Feld and D. Goncalves, Scalar simplified models for dark matter, Phys. Rev. D 91 (2015) 015017 [arXiv: 1410.6497] [INSPIRE].

[20] A. Berlin, S. Gori, T. Lin and L.-T. Wang, Pseudoscalar portal dark matter, Phys. Rev. D 92 (2015) 015005 [arXiv: 1502.06000] [INSPIRE].

[21] T. Jacques and K. Nordström, Mapping monojet constraints onto simplified dark matter models, JHEP 06 (2015) 142 [arXiv:1502.05721] [INSPIRE].

[22] M. Garny, A. Ibarra and S. Vogl, Signatures of Majorana dark matter with t-channel mediators, Int. J. Mod. Phys. D 24 (2015) 1530019 [arXiv:1503.01500] [inSPIRE].

[23] M. Chala, F. Kahlhoefer, M. McCullough, G. Nardini and K. Schmidt-Hoberg, Constraining dark sectors with monojets and dijets, JHEP 07 (2015) 089 [arXiv: 1503.05916] [INSPIRE].

[24] N.F. Bell, Y. Cai, J.B. Dent, R.K. Leane and T.J. Weiler, Dark matter at the LHC: effective field theories and gauge invariance, Phys. Rev. D 92 (2015) 053008 [arXiv:1503.07874] [INSPIRE].

[25] M.A. Shifman, A.I. Vainshtein and V.I. Zakharov, Remarks on Higgs boson interactions with nucleons, Phys. Lett. B 78 (1978) 443 [InSPIRE].

[26] G. Bélanger, F. Boudjema, A. Pukhov and A. Semenov, Dark matter direct detection rate in a generic model with MicrOMEGAs 2.2, Comput. Phys. Commun. 180 (2009) 747 [arXiv:0803.2360] [INSPIRE].

[27] P. Junnarkar and A. Walker-Loud, Scalar strange content of the nucleon from lattice QCD, Phys. Rev. D 87 (2013) 114510 [arXiv:1301.1114] [INSPIRE].

[28] H.-Y. Cheng and C.-W. Chiang, Revisiting scalar and pseudoscalar couplings with nucleons, JHEP 07 (2012) 009 [arXiv: 1202.1292] [INSPIRE].

[29] R.J. Hill and M.P. Solon, Standard model anatomy of WIMP dark matter direct detection I: weak-scale matching, Phys. Rev. D 91 (2015) 043504 [arXiv:1401.3339] [INSPIRE].

[30] R.J. Hill and M.P. Solon, Standard model anatomy of WIMP dark matter direct detection II: QCD analysis and hadronic matrix elements, Phys. Rev. D 91 (2015) 043505 [arXiv: 1409.8290] [INSPIRE].

[31] D. Diakonov, M.V. Polyakov and C. Weiss, Hadronic matrix elements of gluon operators in the instanton vacuum, Nucl. Phys. B 461 (1996) 539 [hep-ph/9510232] [INSPIRE].

[32] M. Felizardo et al., Final analysis and results of the phase II SIMPLE dark matter search, Phys. Rev. Lett. 108 (2012) 201302 [arXiv:1106.3014] [INSPIRE].

[33] COUPP collaboration, E. Behnke et al., First dark matter search results from a $4 \mathrm{~kg} \mathrm{CF} \mathrm{C}_{3} \mathrm{I}$ bubble chamber operated in a deep underground site, Phys. Rev. D 86 (2012) 052001 [arXiv: 1204.3094] [INSPIRE].

[34] J. Goodman, M. Ibe, A. Rajaraman, W. Shepherd, T.M.P. Tait and H.-B. Yu, Constraints on dark matter from colliders, Phys. Rev. D 82 (2010) 116010 [arXiv:1008.1783] [InSPIRE].

[35] Y. Bai, P.J. Fox and R. Harnik, The Tevatron at the frontier of dark matter direct detection, JHEP 12 (2010) 048 [arXiv: 1005.3797] [InSPIRE]. 
[36] CMS collaboration, Search for dark matter, extra dimensions and unparticles in monojet events in proton-proton collisions at $\sqrt{s}=8$ TeV, Eur. Phys. J. C 75 (2015) 235 [arXiv: 1408.3583] [INSPIRE].

[37] ATLAS collaboration, Search for new phenomena in monojet plus missing transverse momentum final states using $10 \mathrm{fb}^{-1}$ of pp collisions at $\sqrt{s}=8$ TeV with the ATLAS detector at the LHC, ATLAS-CONF-2012-147, CERN, Geneva Switzerland (2012) [ATLAS-COM-CONF-2012-190].

[38] A. Alloul, N.D. Christensen, C. Degrande, C. Duhr and B. Fuks, FeynRules $2.0-a$ complete toolbox for tree-level phenomenology, Comput. Phys. Commun. 185 (2014) 2250 [arXiv:1310.1921] [INSPIRE].

[39] J. Alwall, M. Herquet, F. Maltoni, O. Mattelaer and T. Stelzer, MadGraph 5: going beyond, JHEP 06 (2011) 128 [arXiv:1106.0522] [INSPIRE].

[40] T. Sjöstrand, S. Mrenna and P.Z. Skands, A brief introduction to PYTHIA 8.1, Comput. Phys. Commun. 178 (2008) 852 [arXiv:0710.3820] [INSPIRE].

[41] J.S. Conway, Pretty Good Simulation of high-energy collisions, 090401 release.

[42] CMS collaboration, Search for invisible decays of Higgs bosons in the vector boson fusion and associated ZH production modes, Eur. Phys. J. C 74 (2014) 2980 [arXiv:1404.1344] [INSPIRE].

[43] ATLAS collaboration, Search for invisible decays of a Higgs boson using vector-boson fusion in pp collisions at $\sqrt{s}=8 \mathrm{TeV}$ with the ATLAS detector, arXiv:1508.07869 [INSPIRE].

[44] Y. Bai, V. Barger, L.L. Everett and G. Shaughnessy, Two-Higgs-doublet-portal dark-matter model: LHC data and Fermi-LAT 135 GeV line, Phys. Rev. D 88 (2013) 015008 [arXiv: 1212.5604] [INSPIRE].

[45] U. Haisch and E. Re, Simplified dark matter top-quark interactions at the LHC, JHEP 06 (2015) 078 [arXiv:1503.00691] [INSPIRE].

[46] Y. Bai and B.A. Dobrescu, Heavy octets and Tevatron signals with three or four b jets, JHEP 07 (2011) 100 [arXiv: 1012.5814] [INSPIRE].

[47] R.S. Chivukula, A. Farzinnia, J. Ren and E.H. Simmons, Constraints on the scalar sector of the renormalizable coloron model, Phys. Rev. D 88 (2013) 075020 [arXiv:1307.1064] [INSPIRE].

[48] A.V. Manohar and M.B. Wise, Modifications to the properties of the Higgs boson, Phys. Lett. B 636 (2006) 107 [hep-ph/0601212] [INSPIRE].

[49] U. Aglietti, R. Bonciani, G. Degrassi and A. Vicini, Analytic results for virtual QCD corrections to Higgs production and decay, JHEP 01 (2007) 021 [hep-ph/0611266] [INSPIRE].

[50] R. Boughezal and F. Petriello, Color-octet scalar effects on Higgs boson production in gluon fusion, Phys. Rev. D 81 (2010) 114033 [arXiv:1003.2046] [INSPIRE].

[51] Y. Bai, J. Fan and J.L. Hewett, Hiding a heavy Higgs boson at the 7 TeV LHC, JHEP 08 (2012) 014 [arXiv: 1112.1964] [INSPIRE].

[52] B.A. Dobrescu, G.D. Kribs and A. Martin, Higgs underproduction at the LHC, Phys. Rev. D 85 (2012) 074031 [arXiv: 1112.2208] [INSPIRE].

[53] ATLAS and CMS collaborations, Measurements of the Higgs boson production and decay rates and constraints on its couplings from a combined ATLAS and CMS analysis of the LHC pp collision data at $\sqrt{s}=7$ and 8 TeV, ATLAS-CONF-2015-044, CERN, Geneva Switzerland (2015). 
[54] M.I. Gresham and M.B. Wise, Color octet scalar production at the LHC, Phys. Rev. D 76 (2007) 075003 [arXiv: 0706.0909] [INSPIRE].

[55] Y. Bai, A. Katz and B. Tweedie, Pulling out all the stops: searching for RPV SUSY with stop-jets, JHEP 01 (2014) 040 [arXiv:1309.6631] [INSPIRE].

[56] CMS collaboration, Search for pair-produced resonances decaying to jet pairs in proton-proton collisions at $\sqrt{s}=8 \mathrm{TeV}$, Phys. Lett. B 747 (2015) 98 [arXiv:1412.7706] [INSPIRE].

[57] CMS collaboration, Search for new physics in the multijet and missing transverse momentum final state in proton-proton collisions at $\sqrt{s}=8 \mathrm{TeV}$, JHEP 06 (2014) 055 [arXiv: 1402.4770] [INSPIRE].

[58] D. Goncalves-Netto, D. Lopez-Val, K. Mawatari, T. Plehn and I. Wigmore, Sgluon pair production to next-to-leading order, Phys. Rev. D 85 (2012) 114024 [arXiv:1203.6358] [INSPIRE].

[59] T. Appelquist, H.-C. Cheng and B.A. Dobrescu, Bounds on universal extra dimensions, Phys. Rev. D 64 (2001) 035002 [hep-ph/0012100] [INSPIRE].

[60] M. Perelstein, Little Higgs models and their phenomenology, Prog. Part. Nucl. Phys. 58 (2007) 247 [hep-ph/0512128] [INSPIRE].

[61] S. Weinberg, Larger Higgs exchange terms in the neutron electric dipole moment, Phys. Rev. Lett. 63 (1989) 2333 [INSPIRE].

[62] CMS collaboration, Search for vector-like charge 2/3T quarks in proton-proton collisions at $\sqrt{s}=8 \mathrm{TeV}$, arXiv:1509.04177 [INSPIRE].

[63] CMS collaboration, Inclusive search for a vector-like $T$ quark with charge $2 / 3$ in pp collisions at $\sqrt{s}=8 \mathrm{TeV}$, Phys. Lett. B 729 (2014) 149 [arXiv:1311.7667] [InSPIRE].

[64] S. Hoeche et al., Matching parton showers and matrix elements, hep-ph/0602031 [INSPIRE].

[65] LUX collaboration, D.S. Akerib et al., First results from the LUX dark matter experiment at the Sanford Underground Research Facility, Phys. Rev. Lett. 112 (2014) 091303 [arXiv: 1310.8214] [INSPIRE].

[66] SIMPLE collaboration, M. Felizardo et al., The SIMPLE phase II dark matter search, Phys. Rev. D 89 (2014) 072013 [arXiv: 1404.4309] [INSPIRE].

[67] PICASSO collaboration, S. Archambault et al., Constraints on low-mass WIMP interactions on ${ }^{19} \mathrm{~F}$ from PICASSO, Phys. Lett. B 711 (2012) 153 [arXiv:1202.1240] [INSPIRE].

[68] PICO collaboration, C. Amole et al., Dark matter search results from the PICO-2L $\mathrm{C}_{3} F_{8}$ bubble chamber, Phys. Rev. Lett. 114 (2015) 231302 [arXiv: 1503.00008] [INSPIRE].

[69] ICECube collaboration, M.G. Aartsen et al., Search for dark matter annihilations in the sun with the 79-string IceCube detector, Phys. Rev. Lett. 110 (2013) 131302 [arXiv:1212.4097] [INSPIRE].

[70] Super-Kamiokande collaboration, K. Choi et al., Search for neutrinos from annihilation of captured low-mass dark matter particles in the sun by Super-Kamiokande, Phys. Rev. Lett. 114 (2015) 141301 [arXiv:1503.04858] [INSPIRE].

[71] R.M. Godbole, G. Mendiratta and T.M.P. Tait, A simplified model for dark matter interacting primarily with gluons, JHEP 08 (2015) 064 [arXiv:1506.01408] [INSPIRE]. 